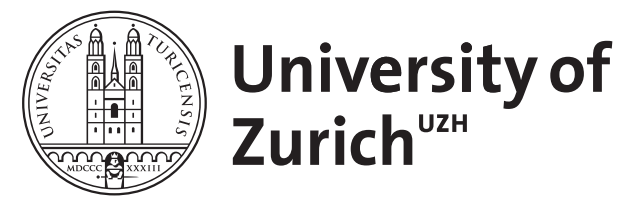

\title{
Die Verfügbarkeit des Teufels und die Kunst
}

\author{
Schnyder, Mireille
}

\begin{abstract}
The article demonstrates how art and technique in the patristic discourse of antiquity are interpreted as strategies of making things verfügbar and associating them with the devil: this is shown in two image conceptions of Christian-theological discourse which are embodied in an exemplary way, on the one hand, by the idolater and tyrannical world conqueror Ninus and, on the other, by the dominator of demons, Zoroaster (Zarathustra). A central hypothesis is that those image reflections which are shaped following a Christian model are not determined by the interest in their production: they focus on the receptional level on which art can be effective as the object of a specific perception ad malam or ad bonam partem.
\end{abstract}

DOI: https://doi.org/10.1524/para.2012.0024

Posted at the Zurich Open Repository and Archive, University of Zurich

ZORA URL: https://doi.org/10.5167/uzh-76163

Journal Article

Published Version

Originally published at:

Schnyder, Mireille (2012). Die Verfügbarkeit des Teufels und die Kunst. Paragrana, 21(2):47-59.

DOI: https://doi.org/10.1524/para.2012.0024 


\section{Die Verfügbarkeit des Teufels und die Kunst}

The article demonstrates how art and technique in the patristic discourse of antiquity are interpreted as strategies of making things verfügbar and associating them with the devil: this is shown in two image conceptions of Christian-theological discourse which are embodied in an exemplary way, on the one hand, by the idolater and tyrannical world conqueror Ninus and, on the other, by the dominator of demons, Zoroaster (Zarathustra). A central hypothesis is that those image reflections which are shaped following a Christian model are not determined by the interest in their production: they focus on the receptional level on which art can be effective as the object of a specific perception ad malam or ad bonam partem.

Wenn man von der Geschichte des Sündenfalls absieht, in der der Teufel als Verführer der Menschen ins Spiel gebracht ist, tritt der Teufel da in die Welt, wo Kunst und Technik sind. Ob bei Tertullian, bei Cyprian, Augustinus oder anderen, die böswilligen Dämonen, die nichts anderes wollen, als die Menschen in ihre Gottferne hereinzuholen, finden da, wo Statuen und Bilder verehrt werden, eine Möglichkeit des Wirkens und Einwirkens. Denn, wie Tertullian - in Übereinstimmung unter anderem mit Laktanz - sagt, hat ,der Teufel Bildhauer, Maler und Verfertiger von Bildnissen aller Art in die Welt gesetzt", und es sind die Hände der Handwerker und Künstler, die den Dämonen Leiber verleihen; ${ }^{1}$ ja, er spricht davon, dass die Künstler den Teufel und seine Engel fabrizieren, deren ganze Götterwürde auf ihrer Geschicklichkeit basiert. ${ }^{2}$ Diese radikale Bild-, Kunst- und Handwerkskritik des strengen Tertullian holt ihre Argumente aus dem antiken Kunstdiskurs einerseits, steht aber andererseits auch in der Tradition eines heilsgeschichtlichen Kunst-Narrativs. Im Folgenden versuche ich aufgrund dieser christlichen Deutungsmuster zwei Bildkonzeptionen herauszuarbeiten, in denen in je eigener Art das Teuflische eine Rolle spielt. Handwerk, Technik und Wissen, alles, was es für die Verfertigung von Kunst braucht, ist eng an die verfluchten Söhne Adams gebunden. Denn während das Geschlecht von Seth, der an die Stelle des ermordeten Abel tritt, alle Gottesverehrer hervorbringt (Enoz, Noah, Abraham), ist es das Geschlecht Kains, das Ackerbau, Nähen, Jagen, Schmiedekunst und Musik erfindet, und das Geschlecht des verfluchten Noah-Sohns Cham, das dieses Wissen

1 Tertullian: Über den Götzendienst/De Idolatria, Abschnitt 7, S. 146. Zu Laktanz siehe Lactance: Institutions Divines, Livre II, Kapitel 16,1-3, S. 194f. Hinweis bei Pekáry 2002, S. 92.

2 Tertullian, Über den Götzendienst, Abschnitt 6, S. 145. 
nach der Sintflut wieder aufnimmt. ${ }^{3}$ Die menschlichen Künste gehören so von Anfang an zu einer Gottferne und bleiben darauf bezogen.

\title{
I. Das Götzenbild
}

Rudolf von Ems erzählt in seiner Weltchronik die im Mittelalter und in der Spätantike sehr bekannte Geschichte von Ninus, dem ersten Tyrannen der Weltgeschichte (als der er topisch in die Historiographie eingegangen ist) und auch ,Erfinder" der Götzenbilder: ${ }^{4}$ Ninus, der seinen Vater maßlos liebte, ließ nach dessen Tod von ihm eine Statue machen, um ihn weiterhin vor sich sehen zu können.

\author{
[Er] hiez mit vil richin sachin \\ ein schone bilde machin, \\ das sinim vatir was gelich, \\ und hiez das machin alse rich \\ alse kúnegis richeit wol gezam. (V. 3436-3440)
}

[Er] ließ mit viel kostbaren Materialien / ein schönes Bild herstellen, / das seinem Vater gleich sah / und er ließ es so kostbar machen, / wie es der Macht eines Königs angemessen war.

Die Schönheit dieses Bildes ist definiert über die Kostbarkeit des Materials und die Ähnlichkeit mit dem Dargestellten sowohl in Bezug auf die individuelle Erscheinungsform des Vaters als auch auf dessen Wert in der gesellschaftlichen Ordnung.

Der Anblick dieses Bildes bewirkt bei Ninus dieselbe Freude, wie er sie in der Gegenwart des Vaters empfunden hatte (V. 3447-3450), so dass er seine Trauer vergisst:

und wart der angesiht so vro

das er virgaz von liebi gar

swas im ê herzeleidis war. (V. 3451-3453)

und er wurde so glücklich über den Anblick, / dass er aus lauter Liebe vergaß, / was immer ihm vorhin noch Herzenskummer bereitet hatte.

Durch die Identität des Affekts gegenüber dem Vater und dem Bild ergibt sich eine Illusion der Präsenz des Abwesenden/Verstorbenen. Und darüber wird, in der Wahrnehmung des Bildes, der Tod des Vaters vergessen, das Vergangene negiert und - in der Scheinpräsenz - die Zeitwahrnehmung zerstört. Es ist eine Stillstellung des Moments, ein Einfrieren eines Affekts im reglosen, unbeweglichen Bild.

3 Vgl. zu dieser Vorstellung u. a. Augustinus: Vom Gottesstaat, 15. Buch, 21. Kapitel, S. 260-262. Vgl. auch die Darstellung bei Rudolf von Ems: Die Weltchronik, V. 475-618, S. 7-9, sowie die Quelle von Rudolf: Petrus Comestor: Historia Scholastica, lib. Gen., Cap. 28, Sp. 1078f.

4 Vgl. zu Ninus als Tyrannen: Rudolf von Ems, Weltchronik, V.3410-3419, S. 48. Interessant ist, dass in der Antike negativ dargestellten Kaisern oft Bildhauerei und Malerei zugeschrieben wurden. Vgl. dazu Pekáry 2002, S. 80. 
Dieses persönliche und private Totengedenken des Sohnes wird dann aber, wegen seiner öffentlichen Rolle als König, durch andere nachgeahmt, indem jeder seinen ihm liebsten Freund nach dessen Tod ins Bild setzt. So installiert sich im ganzen Reich ein Totenkult: der sitte begunde für sih gan / und wart als ellich ubirz lant (V.3471f.; „Der Brauch breitete sich aus und wurde so gemein im ganzen Land"). Schließlich kommt es im Zusammenspiel der Macht des Gewaltherrschers und der Einfalt des Volkes dazu, dass die Erinnerungsgeste zu einer Anbetungsgeste wird und der im Abbild aus der Vergangenheit in die sichtbare Präsenz herausgelöste (geliebte) Mensch als ein gegenwärtiger Gott erscheint:

\author{
disú ere und dirre site \\ gedech darzů das wip und man \\ fúr got dú bilde beten an: \\ wand das lút einvaltig \\ was und der man gewaltic \\ der die site uz brahte. (V. 3475-3480)
}

Diese Verehrung und dieser Brauch / gediehen so weit, dass Frau und Mann / die Bilder als Gott anbeteten: / denn das Volk war einfältig / und der Mann mächtig, / der diesen Brauch installierte.

Über die Bilder installiert sich eine Göttergesellschaft, in der sich die weltliche spiegelt. Denn: der rihin bilde waren rih, / der armin als sis mohtin han (V.3469f.; „Die Bildnisse der Vornehmen waren kostbar und die der Armen so, wie sie es vermochten"). Und das durch den emotionalen Bezug individualisierende Bild konstituiert über die Vervielfältigung ein System des die Materialität transzendierenden Begehrens. Dabei wirkt eine doppelte Mimesis: Einerseits ist es die Nachahmung der Wirklichkeit in der Ähnlichkeit des Bildnisses mit dem Verstorbenen, erreicht durch das handwerkliche Geschick der Künstler und die Qualität des Materials, anderseits ist es die Nachahmung der königlichen Handlung durch das Volk, worüber sich im Machtbereich des Tyrannen der Kult institutionalisiert. ${ }^{5}$ Die Kunst als Mittel der Wahrnehmungstäuschung und Illusion wird über die Praxis der Macht zum Mittel eines kollektiven Imaginären. Zielt das eine auf Stimulierung individualisierender Emotionen, über die der rationale Blick auf das Bild geblendet wird und jede Reflexion verunmöglicht - wie das auch im Kunstdiskurs der Antike Thema ist $^{6}-$, dient das andere der Verankerung der Macht und ihrer Ordnung in der Transzendenz.

Das Kunstwerk, auf Illusion angelegt, verstellt den Blick auf ein in Zeit- und Raum-Kategorien fassbares Reales und provoziert eine Emotionalität, die sich dem rationalen Zugriff entzieht. So hat denn nach dem Text von Rudolf von Ems - der aber darin gut abgesichert ist durch die Schriften der Kirchenväter - auch genau hier der Teufel seine Finger im Spiel.

5 Diese kausale Verknüpfung von Installation eines heidnischen Kultes und Macht des Herrschers ist topisch: Es ist immer der Tyrann, der den Götzenkult dem Volk auferlegt.

6 Vgl. zu dieser Vorstellung die antike Kunstpolemik. Dazu Pekáry 2002, S. 20, sowie weiter unten in diesem Beitrag. 


\section{I.1 Der Teufel im Bild}

durh des túvils spot (V.3485; „,zum Vergnügen des Teufels“ / „wegen des Vergnügens des Teufels") beginnen die Leute ihre Ahnen, Heroen und Geliebten als Götter zu verehren. Die über das kunstvolle Bildwerk angestachelte Emotion, als Trost in der Scheinpräsenz und Liebe zur Fälschung, wird zum Ursprung und Motiv der teuflisch angestachelten Transzendierung des Kunstwerks und Abkehr von der Wirklichkeit/Wahrheit. Genau in dem Moment, wo das Bild seinen Abbildcharakter verliert, die Memoria zur Anbetung wird, Zeitstruktur und Raumstruktur sich auflösen, die Individualität des liebenden Erinnerungsbezugs zu einem verallgemeinerbaren, verehrenden Gottesbezug wird, ist die Materie des Bildes über die Wahrnehmung des Menschen so zugerichtet, dass sich der Teufel darin verkörpern kann:

Im Vergessen der Bedingungen der Kunst, der Materialität und Technizität, inszeniert sich der Teufel. ${ }^{7}$ Die Teufelsmacht des Bildes ist ein Rezeptionsproblem.

als mans an bette und eren began,

so für der túvil drin zehant

und tet daruz antwúrte irchant,

und half iedanne sa dem man

darumbe er in bette an:

wand swes der man was vollekomen,

darubir wart er ze gote gnomen,

darumbe im ere wart irboten.

der tievil gap dén abgoten

die ere und ouh ir helfe kraft

das si nah sinir meistirschaft

das lút kundin beratin,

swar ubir si helfe batin. (V. 3503-3515)

Als man es [das Bildnis] anzubeten und zu verehren begann, / fuhr der Teufel sofort darein / und gab daraus Antwort / und half dann jedem Menschen, / worum er zu ihm betete. / Denn worin ein Mensch vollkommen war, / dafür wurde er zum Gott erhoben, / dafür wurde ihm Ehre erboten. / Der Teufel gab den Götzen / das Ansehen und auch die Potenz zu helfen, / so dass sie gemäß seiner Herrschaft (seinem Können) / die Leute beraten konnten / worum sie um Hilfe baten.

Die Ambivalenz zwischen teuflischer Machenschaft, menschlichem Kunstgebrauch und tyrannischer Machtwirkung, die hier mit im Spiel ist, zeigt sich an der Namensgebung der neu installierten Götter.

wand der [der Vater des Ninus] Belus was genant,

do lerte si des túvils spot,

das man dú hohsten abgot

der man do pflag, sus hiez nahim:

Bel, Baal und Baalim

7 Ganz ähnlich heisst es bei Marcus Minucius Felix: tunc postremo deus est, cum homo illum voluit et dedicavit. (,Jetzt endlich ist er ein Gott, da ein Mensch ihn so wollte und weihte"). Octavius, Kapitel 24,8, S. 138f. 
und Belzebub, der nah der art

ein túvil sit geheizin wart,

der fúrste manegir túviln ist. (V. 3491-3498)

„Da er [der Vater des Ninus] Belus genannt wurde, / lehrte sie der Spott des Teufels, / dass man die höchsten Götter, / die man da verehrte, so nach ihm nannte: / Bel, Baal und Baalim / und Belzebub, der entsprechend seiner Art / seither ein Teufel genannt wurde / der der Fürst von vielen Teufeln ist.“

Indem der höchste Gott nach dem Vater des Ninus Belus genannt wird, kann er in heilsgeschichtlich-christlicher Namensmagie mit dem Höllenfürsten identifiziert werden. Über die Metamorphose des Namens wandelt sich so der Vaterkult zum Teufelskult und die Götterwelt, die die weltliche Gesellschaftshierarchie spiegelt, wird zur Teufelsgesellschaft. Da, wo es zu einer sinnstiftenden Diversifizierung über die Bezeichnung kommt, hat der Teufel die Hände im Spiel. ${ }^{8}$ Damit ist die Götterordnung von Anfang an nominell an die Hierarchie der Teufel gebunden.

\section{I.2 Teuflische Machenschaften: Das Leben des Bildes}

Der Teufel kann sich aber erst dann ins Bild schmiegen, wenn das Kunstwerk wirkt. Minucius Felix lässt den Christen im Octavius (2./3. Jh.) sagen, dass „der Verstand der Unwissenden getäuscht ist durch die Perfektion der Kunst, geblendet durch das Glänzen des Goldes, verdunkelt durch das Leuchten des Silbers und die Weisse des Elfenbeins“". ${ }^{9}$ Erst wenn das Kunstwerk den Moment stillstellt, die Zeit der Vergangenheit zur Gegenwart macht und diese auf Dauer setzt, so dass der Betrachter nicht mehr reflektiert, sondern die Materie transzendiert, erst dann ist der Moment für den Teufel gekommen, sich das Bildwerk als Körper anzuziehen. Und dann (mit Hilfe des Teufels) transzendiert das Kunstwerk seine Bedingung, indem es sich belebt: Es spricht, handelt, berät, heilt, wirkt - dies in einem Raum der totalen Illusion.

Dabei fällt auf, dass in diesem Narrativ, sei es bei den Kirchenvätern, sei es in den heilsgeschichtlichen Erzählungen, Bildkunst immer nur als Götzenbild konzi-

8 Vgl. M. Minucius Felix, Octavius, Kap. 18,10, S. 108f.: „Suche nicht nach einem Namen für Gott: ,Gott‘ ist sein Name. Nur da braucht man Benennungen, wo man eine Vielzahl durch die besonderen Eigenschaften der Bezeichnung in Einzelnes unterteilen muß; Gott aber, der einzig ist, gehört die Benennung ,Gott‘ ganz und gar zu eigen." (Nec nomen deo quaeras: Deus nomen est. illic vocabulis opus est, cum per singulos propriis appellationum insignibus multitudo dirimenda est: deo, qui solus est, Dei vocabulum totum est.). Verunklärt bleibt aber das präzise Verhältnis von Namensgeber, Namensträger und Gottesname. Zwar lerte si des tuvels spot (V.3493; „gab ihnen die teuflische Gemeinheit ein"), den höchsten Gott Bel zu nennen, doch ist nicht klar, nach wem der Name sich richtet: nach im, dem Teufel, oder nach im, dem Vater Belus? Ist des Teufels Name von Anfang an schon im Namen des Vaters, oder ist der Vatername der Namensgeber für den Teufel?

9 M. Minucius Felix, Octavius, Kap. 24,5, S. 136f.: Quis ergo dubitat horum imagines consecratas vulgus orare et publice colere, dum opinio et mens imperitorum artis concinnitate decipitur, auri fulgore praestringitur, argenti nitore et candore eboris hebetatur? 
piert ist. Oder anders: Es gibt kein Bild außer dem Götzenbild. Am schärfsten findet sich dieser Gedanke wieder bei Tertullian, der im Prinzip jedes Handwerk der Idolatrie bezichtigt, da es in der Potenz den Götzenbildern dient. Da ist es selbst gefährlich, Schuhmacher zu sein. ${ }^{10}$

Damit wird an die Kunstkritik der Antike angeknüpft, die die Wirkmacht der Kunst im Sinne einer wahrnehmungstäuschenden Stimulierung von Emotionen (vor allem des Begehrens) reflektiert. Zeugnis davon geben die unzähligen Geschichten von geliebten, geküssten und vergewaltigten Statuen. ${ }^{11}$ Huren, Zecher und Bildbetrachter gehören für Sallust zusammen. ${ }^{12}$ Und auch Artemidor sagt in seiner Traumdeutung: „Das Modellieren, Malen, Ziselieren und Verfertigen von Statuen [ist] für Ehebrecher, Rhetoren, Fälscher und alle Betrüger von guter Vorbedeutung, weil diese Künste das nicht Existierende als existierend darstellen. “13 Immer wieder wird so auf den Grund-Betrug der Kunst hingewiesen.

Die Destruktion dieser täuschenden Bildwirkung versucht man - in der antiken Bildkritik, deren Argumente dann in der christlichen Götzenkritik aufgenommen werden - über die Reduzierung des Bildes auf seine Materialität. So wird betont, dass es nichts anderes sei als Farbe und Leinwand, Marmor und Gold, Blech und Silber, ohne Leben, ohne Kraft. Und, in krassem Gegensatz zu der sich genau dafür brüstenden Rhetorik und Dichtung, fehle dem Bild die Möglichkeit, Bewegung oder die belebende Seele einzufangen. ${ }^{14}$ Entsprechend sind belebte Kunstwerke entweder raffinierte Maschinerien oder dann Täuschung, reine Illusion, reiner Schein. ${ }^{15}$ In diesem Sekundärbereich der Schöpfung tummeln sich dann aber auch die Dämonen, die, in der christlichen Lesart zu Teufeln mutierend, immer darauf erpicht sind, die göttliche Schöpfung zu verfälschen, zu pervertieren, zu entstellen. ${ }^{16}$

\section{Das Zauberbild}

Die Geschichte von Ninus ist aber noch nicht zu Ende. In der kleinen Fortsetzung, wie sie die Weltchronik von Rudolf von Ems berichtet, besiegt er, Welteroberer und Götzenerfinder, auf dem Höhepunkt seiner Macht den König Zoroaster.

er sli̊g, als úns dú warheit seit,

einin ediln kúnic riche,

10 Tertullian, Götzendienst, S. 148 u. ö.

11 Vgl. dazu Pekáry 2002, S. 20 und S. 24f.

12 Ebd., S. 23.

13 Artemidor: Traumkunst, Kapitel I,51, S. 67f.

14 Pekáry 2002, S. 53-69. Vgl. auch Longinus: Vom Erhabenen (1988).

15 Der Künstler ist so immer der unfähige Gott. Denn dieser schafft - als bester Künstler - beseelte Statuen. Zum antiken und frühchristlichen Hintergrund dieser Vorstellung vgl. Pekáry 2002, S. 83-85.

16 Der Teufel ist der „Affe Gottes“, wie es Justin Martyr formulierte (Dialogus cum Tryphone Judaeo). Zitiert nach Roskoff 1987, Bd. 1, S. 224. 
des wisheit vil wisliche

zoubirliche liste vant:

der Zoroastres was genant,

ein kúnic bactrianschen diet,

des kunst mit wisheit beschiet

zoubirliche liste funt. (V. 3529-3536)

Er besiegte, wie uns die Wahrheit sagt, / einen mächtigen, edlen König, / dessen Weisheit voller Wissen Zauberkunst erfand: / er war Zoroastres genannt, / ein König des baktrischen Volks, / dessen Kunst, mit Weisheit ausgestattet, Zauberkunst erfand.

Dieser ist - nach der von Rudolf benutzten Tradition - ein baktrischer König, der nicht nur mit Cham (dem Sohn Noahs) gleichgesetzt wird (zumindest nominell), sondern auch als Erfinder der Magie bekannt war. ${ }^{17}$

Die kriegerische Auseinandersetzung zwischen dem tyrannischen Götzendiener Ninus und dem Magier-König Zoroaster ist dadurch, dass sie in der Tradition beide der Linie Chams entstammen und immer wieder in ein Abstammungsverhältnis hineingedacht werden, intrikat.

Magie, als die Kunst über Dämonen zu verfügen, diese in Dienst zu nehmen und zur vielfältigen Manipulation der Materie zu gebrauchen, steht dem tyrannischen Affekt gegenüber, der im Willen der Verstetigung des Moments und im Selbstbezug der Vaterliebe der Illusion einer Überwindung von Zeit, Raum und Materialität zum Opfer fällt und so dem Teufel ein Spielfeld bereitet. Verfügt der eine über mit Weisheit kombinierte Künste, ist der andere von übermäßiger Emotionalität geleitet.

So besiegt in Ninus auch die Macht des den teuflischen Kunstgriffen verfallenen, von Trugbildern umstellten, Bilder anbetenden Tyrannen die Macht des Meisters der freien und mechanischen Künste sowie Erfinders der Magie. ${ }^{18}$ Die Verfügungsgewalt über dämonische Kräfte mit Hilfe von Verstand und Wissen, die Kunst des Zaubers, wird von einer im Dienste der teuflischen Illusion stehenden Macht besiegt.

Damit ist die Kunst des Zaubers aber auch aus dem Interesse der patristischen und theologischen Bildkritik verdrängt. Denn diese bezieht sich auf das Götzenbild, die Idolatrie, und fokussiert hier die Blendung des Verstands, die getäuschte

17 Vgl. die Version der Geschichte von Ninus und Zoroaster, die, wohl auf Eusebios von Caesarea zurückgehend, bei Hugo von St. Victor (1096-1141) zu finden ist (Adnotationes elucidatoriae in Pentateuchon) und durch die Historia scholastica von Petrus Comestor (12. Jh.) verbreitet wurde. Hier werden im von Ninus angegriffenen und besiegten König von Baktrien die beiden Figuren von Cham und Zoroaster zusammengebracht. Vgl. dazu Strausberg 1998, vor allem S. 446-454. Zum Teil heißt es auch, dass sich Cham im Alter Zoroaster nannte. So wird also Cham teilweise höchstpersönlich (nicht nur sein Sohn Chus oder einer seiner Nachfolger) zum Erfinder der Magie und für die septem artes liberales zuständig. Bei Augustinus findet sich dann eine Lesart der Zoroastergeschichte, die dessen Niederlage gegen Ninus als Niederlage der Magie versteht: De civitate Dei, 21. Buch, 14. Kapitel, S. 708.

$18 \mathrm{Zu}$ den artes liberales als dem Fundament der schönen Künste „par leur théorie littéraire (grammaire et rhétorique) et leur théorie des proportions plastiques et sonores (arithmétique et musique)“v vgl. de Bruyne 1946/1975, Bd. 1, S. 209. 
Rezeption. Das Bild ist hier immer potenzieller Teufelskörper. Der einzige Schutz dagegen ist das christliche Wort, das allein den Teufel aus dem Bild vertreiben kann. ${ }^{19}$ Es ist die richtige Kontextualisierung, vor allem der sprachliche Kontext, was die Gefahr im christlichen Bild minimieren und die Rezeption steuern kann.

\section{II.1 Die Kunst im Bild}

Es fällt auf, dass sich in den christlich geprägten Bild-Reflexionen der Blick nicht auf die Kunstproduktion richtet, sondern auf die Kunstrezeption. Die hier möglichen Verfehlungen diskreditieren aber das Kunstwerk an sich. Tertullian nennt die Bildner und Schnitzer „Toren, welche Dinge bilden, wie es ihnen beliebt und den Leuten nichts nützt. ${ }^{\text {“20 }}$ Dabei steckt in der - in dieser Aussage mit angeprangerten - Willkür auch die Problematik des sich zum alter creator aufschwingenden Künstlers, der in seinem Anspruch schon Opfer einer teuflischen Hybris ist.

Die Kunstfertigkeit als handwerkliches Geschick, als Beherrschung von Material und konzeptuelle Kraft im Sinne einer mimetischen Erschließung natürlicher Potenzen interessiert aber nicht. ${ }^{21}$ Und wenn, dann ist es in den Kontext der Magie und Zauberei gestellt, die ihrerseits immer stärker aus dem erlaubten christlichen Handlungswissen ausgeklammert wird. Dass Technik, handwerkliches Geschick und Wissen dazu befähigen können, über dämonische Kräfte zu verfügen und mit deren Hilfe Materie und Wirklichkeit zu manipulieren, hat im Verständnis christlicher Kunst keinen Ort. Dass Dämonen anders als über das christliche Heilswort gebannt werden können, ist nicht denkbar.

Und trotzdem gibt es die Tradition des Zarathustra. Und auch wenn Ninus dessen Bücher verbrannt hat, ist sein Wissen überliefert, da Zoroaster - wie vor ihm schon die Nachkommen Kains vor der Sintflut ihr technisches Wissen - die septem artes liberales auf 14 Säulen aufgeschrieben hatte: sieben aus Bronze und sieben aus Ziegel, damit sie auch im Falle einer Sintflut oder eines Weltenbrands erhalten blieben. ${ }^{22}$ Es ist keine Idolatrie, die damit praktiziert wird, es ist auch keine teuflische Machenschaft im Kleid der Künste, sondern es ist die letztlich innerweltliche Kunst der Manipulation, geleitet von wîsheit, list und kunst.

\section{II.2 Kunstgriffe: Gefügige Teufel}

Vor diesem skizzierten Hintergrund wird verständlich, warum in der mittelalterlichen Literatur alle großen Kunstwerke im außerchristlichen Bereich angesiedelt

19 Vgl. unten Anm. 27.

20 Tertullian, Götzendienst, S. 142f.

21 Zum Verständnis von Naturähnlichkeit und Nachahmung der Natur vgl. Speer 1994, vor allem S. 953f.

22 Dazu Strausberg 1998. Zur Tradition der Verbrennung magischer Bücher vgl. Roskoff 1987, S. 288. 
sind und mit Hilfe magischer Mächte hergestellt wurden. Das höchste Lob, das da dann ausgesprochen werden kann, ist: ,als wenn es leben würde“. ${ }^{23}$ Die Perfektion der Wahrnehmungstäuschung ist die Belebung des Kunstwerks, sei es mit mechanischen oder illusionistischen Mitteln. Beides gehört aber in den Bereich eines schon in der Antike unzählige Anekdoten hervorrufenden Kunstverständnisses, dessen Grenze die Lebendigkeit ist und dessen Ziel es ist, diese Grenze zumindest im ,als wenn" zu überschreiten. ${ }^{24}$

Das Grabmal von Blanscheflur in Konrad Flecks Flore-Roman (um 1220), ${ }^{25}$ angelegt als großer Betrug von den Eltern des Floris, um ihn von der Liebe zur Christin Blanscheflur zu befreien, zeigt dies sehr deutlich: Das in der âventiure überlieferte, vom Hörensagen dem Erzähler bekannte (als ich es vernomen habe, V.1990) Kunstgebilde zeichnet sich dadurch aus, dass die daran angebrachten Tierbilder (Löwen, Vögel sowie alles Getier, das auf der Erde nur je lebte und lebt) so sind, wie wenn sie lebten:

\author{
dâ lâgen under \\ vier lewen êrîn, \\ rehte sam sie lebetîn. (V. 1954-1956)
}

Da lagen darunter / vier eherne Löwen, / genau so, wie wenn sie leben würden.

Und:

diu selben bilde

diuhten iuch sô lobelich

daz ir swüert sie regeten sich

und daz sie lebeten garwe. (V. 1968-1971)

Diese Bilder / würden Euch so lobenswert scheinen, / dass ihr schwören würdet, / sie bewegten sich / und wären ganz lebendig.

Dabei wird jedoch schon mit dem diuhten iuch auf die Scheinbarkeit dieser Lebendigkeit hingewiesen, deren Kunstfertigkeit und raffinierte Technik dadurch aber ausgestellt wird. Das wird dann explizit gemacht durch die Erklärung, wie es zu dieser Belebung kommt:

daz kam von maneger varwe

und von des goldes rîchheit. (V. 1972f.)

Das kam von vielfältigen Farben / und von der Kostbarkeit des Goldes.

Und wenn die Sonne schien, blendete der dadurch vom Schmiedewerk zurückgeworfene Glanz die genau hinschauenden Augen.

23 Vgl. dazu Niehr 2003; Fasbender 1999; Belkin 1971, S. 325-346.

24 Vgl. dazu Kris/Kurz 1995, S. 89-99.

25 Konrad Fleck: Flore und Blanscheflur (1846 [Sommer]). 
daz kam von vier sachen,

an die nieman kan gemachen

schoniu bilde cleine:

golt, silber, guot gesteine,

schoniu varwe dez vierde. (V. 1983-1987)

Das kam von vier Dingen, / ohne die niemand / feine, schöne Bilder machen kann: / Gold, Silber, edle Steine, / und als viertes schöne Farben.

Oben drauf hatten die Handwerker-Künstler (die wercmeister, V.1991) das Bild von Flore und Blanscheflur gesetzt: alsô si lacheten / und als sie samet spilten (V. 1992f.; „wie wenn sie lachen und zusammen spielen würden“). Jeder, der sie sah, erkannte die Übereinstimmung mit den Vorbildern.

Diese mit größter Kostbarkeit errichtete Anlage ist an den vier Ecken durch zouberworte der meister (V.2020) so zugerichtet, dass die wehenden Winde durch die Vorrichtung im Inneren bis zu den Kinderbildern geleitet werden, so dass das schone gesmîde sprach / in lebender liute wîse (V.2026f.; ,das schöne Schmiedewerk in der Art lebender Menschen sprach"). Von zouberlisten daz geschach (V.2025), heißt es klar, und die Anlage ist von zwei Schmieden gefertigt, die sich durch ihren Namen an die antike heidnische Tradition anlehnen: Vulcân und Orphanus (V.2029). ${ }^{26}$

Die Beschreibung ist darauf erpicht, einerseits die täuschende Wirkung des Bildes hervorzuheben, anderseits aber die Materialität und Technizität des Werks auszustellen. So wird nicht nur, wie gesagt, auf das Funktionieren hingewiesen (daz kam von ..., V. 1983), sondern wenn die zwei Statuen miteinander reden (in lebender liute wîse, V.2027), spricht einerseits Flôren bilde (V.2030), andererseits antwortet daz ander golt (V.2036).

Dieses kunstreiche Verfügen über das Material und die Naturkräfte (Licht, Wind) findet sich auch in den anderen großen Kunstwerken des nicht-christlichen Raums in der Literatur. So auch im Grabmal der Camilla im Eneasroman Heinrichs von Veldeke. Es ist ein Gebäude (ein geworke, V.251,37) so herrlich und schön, dass man noch nie von Ähnlichem gehört hat.

daz meisterde Gêometras.

des lônde im Kamille.

dar zûu was gût sîn wille,

sint daz hers begonde,

wander wole konde

die list von gêometrîen.

des hôrde ich in zîen,

daz wart dâ an werke schîn,

esn mohte anders niht sîn. (V. 251,40-252,8)

26 Zu der Bedeutung dieser zwei Namen im Kontext von Technik und Zauberei, Schmiedekunst und Magie vgl. den auch sonst für den hier behandelten Zusammenhang aufschlussreichen Artikel von Belkin (1971). 
Das bewerkstelligte Geometras, / was ihm Kamille lohnte. / Darauf richtete er seinen Willen, / seitdem er es begonnen hatte, / denn er beherrschte / die Kunst der Geometrie gut, / das hörte ich von ihm sagen, / das wurde da aber auch im Werk offenbar. / Es hätte nicht anders sein können.

Im Detail wird dann geschildert, wie der wîse man / des werkes zallerêrst began (V.252,9f.; ,wie der weise Mann das Werk allererst anfing“").

Die Steine, die Konstruktion mit Kreuzgewölbe und Säule darauf, alles wird genau beschrieben, wie der wîse man Gêometras, / der des werkes meister was (V.252,23f.; ,der weise Mann Geometras, der der Meister dieses Werkes war“), es machte, und zwar so, daz michel list dar ane schein (V.252,37; „dass man viel Kunst daran erkannte"). Die kostbarsten Materialien und die ausgeklügeltsten Konstruktionen werden beschrieben. Schließlich auch die Balsamfässchen, die den Leichnam nicht vermodern ließen: daz konde wol bedenken / der listige werkman, / der grôz lob dar abe gewan. (V.254,6-8; ,das konnte der kunstvolle Werkmeister gut austüfteln, der großes Lob dafür erntete.“) Höhepunkt ist dann die Installation einer Lampe, deren Docht nie kleiner wurde, gehalten von einer steinernen Taube, beschützt und bedroht von einem Schützen mit gespanntem Bogen, dessen Schuss - nach ausgelöstem Mechanismus - die Taube treffen würde, so dass das Licht erlöschen würde.

ez meisterde ein Crieche
und der wîse Gêometras,
der ein listich man was;
her schûf ez alser wolde.
[...]
nû hôret wie herz ane vienk
der meister, der des alles wielt. (V. 254,38-255,5)

Das bewerkstelligten ein Grieche / und der weise Geometras, / der ein kunstreicher Mann war. / Er schuf es, wie er es wollte. / [...] Nun hört, wie der Meister es anlegte, / der die Aufsicht darüber hatte.

Von dem Spiegel, in dem sich alles sehen ließ im Umkreis von einer Meile, gar nicht zu reden.

Anders als diese letztlich heidnischen Kunstwerke, deren zauberhafte Technik von heidnischen Meistern nach einer gewissen Willkür (alser wolde) bewerkstelligt wird, und die alle in den Texten raffiniert und im Detail beschrieben sind, werden Kunstwerke im christlichen Kontext nie in ihrem Entstehungskontext gezeigt, sondern nur in ihrem Wirkungszusammenhang. Dabei ist das Bildwerk Träger göttlicher Macht, Heilsmedium oder Wunderding.

So ist die Gralsburg, bei der sonst nicht mit Glanz und Inszenierung gespart wird, bildlos - und der Gral selber ist kein Kunstwerk in dem hier beschriebenen Sinn. Wenn schöne Geräte aufgezählt werden, dann mit fremder Herkunft und als Zeichen von Reichtum und Macht. Es gibt keine Künstler, auch keine Handwerker in der Gralswelt. 


\section{Schluss}

Christliche Kunst ist nicht als gemachte Kunst Thema, ist nicht als technisch und kunstfertig hergestellte Illusion interessant, sondern sie interessiert als Objekt einer spezifischen Wahrnehmung, die ad malam und ad bonam partem wirken kann. Das heißt aber, dass Kunst im christlichen Bereich kontextualisiert sein muss, um als göttlich oder teuflisch belebt klassifiziert zu werden.

Es sind diese herkunftslosen, technik- und in einem eigentlichen Sinn kunstlosen Gebilde, über deren Rezeption die göttliche, aber eben auch die teuflische Macht wirken kann. Verfügt der magische Künstler (und damit meine ich den in den artes beschlagenen und in der handwerklichen Ausführung raffinierten Künstler) über die Dämonen, ist es der im christlichen Kontext aus den magischen Fesseln befreite Teufel im verehrten Kunstwerk, der über die Kunst und deren Rezeption verfügt. Zerstört das christliche Wort die Wirkung des Bildes, sei es, dass der Teufel ausgetrieben wird, sei es, dass die Materialität des Bildes auf Gott hin transzendiert wird, ${ }^{27}$ schafft das Zauber-Wort das Bild und damit auch dessen Wirkung, die eng mit der Gemachtheit des Bildes, der Materialität und deren je eigener Konfiguration zusammenhängt.

Diese grundsätzlich andere Art der Bildkonzeption und Bildwahrnehmung, die sich im christlich-theologischen Diskurs und dessen Rezeption zwischen dem Bild in christlichem Kontext und dem Bild in heidnischem Kontext auftut, mag mit ein Grund dafür sein, dass es der abendländischen Kunst möglich wurde, den Künstler - jenseits seiner Handwerksbeherrschung - zu vergöttlichen. Denn da, wo der Entstehungsprozess mit all seinen Wissen, Können und Geschick fordernden Schritten in den Vordergrund rückt, fasziniert die Gewalt des Einzelnen, der souverän, raffiniert und nicht immer nachvollziehbar über die Materie und deren Kräfte verfügt. Da aber, wo die Wirkung eines Bildes Thema ist, ohne dass seine Entstehung weiter bedacht wird, ist das Bild Gefäß für göttliche oder teuflische Machenschaften. Der Urheber des Bildes aber wird dadurch zum göttlich inspirierten oder teuflisch instrumentalisierten Ausführungsorgan. So lässt sich fragen, ob die kunstgeschichtlichen Wertungen, implizit, aber durchaus auch explizit zu finden, die die außereuropäischen Künste in der Regel im Bereich des uninspirierten Handwerklichen oder Ornamentalen ansiedeln, nicht späte Folgen des Siegs des Teufelsanbeters Ninus über den Dämonenbeherrscher Zarathustra sind.

27 Die Vorstellung, dass allein das christliche Wort die in den verehrten Statuen wirkenden Dämonen bannen und die Täuschung entlarven kann, ist topisch. Vgl. M. Minucius Felix, Octavius, Kap. 27,5, S. 156f.: „Wie die meisten von euch wissen, geben die Dämonen das alles über sich selbst auch zu, wenn wir sie mit der Folter der Beschwörung und der Glut unserer Gebete aus den Leibern austreiben“ (Haec omnia sciunt plerique pars vestrum ipsos daemonas de semet ipsis confiteri, quotiens a nobis tormentis verborum et orationis incendiis de corporibus exiguntur). 


\section{Literatur}

\section{Texte}

Artemidor (1991): Traumkunst. Übersetzung von Friedrich S. Krauss, neubearbeitet und mit einem Nachwort sowie Anmerkungen versehen von Gerhard Löwe. Einleitung von Fritz Jürß. Leipzig.

Augustinus: Vom Gottesstaat/De civitate Dei (1995). Aus dem Lateinischen übertragen von Wilhelm Thimme, eingeleitet und kommentiert von Carl Andresen. München.

Heinrich von Veldeke: Eneasroman. Mittelhochdeutsch/Neuhochdeutsch. Nach dem Text von Ludwig Ettmüller ins Neuhochdeutsche übersetzt, mit einem Stellenkommentar und einem Nachwort von Dieter Kartschoke. Stuttgart 1989 (Reclams Universal-Bibliothek 8303).

Konrad Fleck: Flore und Blanscheflur (1846). Eine Erzählung. Hg. von Emil Sommer. Quedlinburg (Bibliothek der gesammten deutschen National-Literatur von der ältesten bis auf die neuere Zeit 12).

Lactance [Laktanz]: Institutions Divines. Livre II: L'origine de l'erreur/Divinarum Institutionum. Liber Secundus: De origine erroris. Introduction, texte critique, traduction et notes par Pierre Monat. Paris 1967 (Sources Chrétiennes 337).

Longinus: Vom Erhabenen. Griechisch/Deutsch. Übersetzt und hg. von Otto Schönburger. Stuttgart 1988 (Reclams Universal-Bibliothek 8469).

Marcus Minucius Felix: Octavius (1965). Lateinisch/Deutsch. Hg., übersetzt und eingeleitet von Bernhard Kytzler. München.

Petrus Comestor: Historia Scholastica (1966). In: PL 198. Turnholt, Sp. 1049-1722.

Rudolf von Ems: Die Weltchronik (1915). Aus der Wernigeroder Handschrift. Hg. von Gustav Ehrismann. 3. Nachdruck der Ausgabe Berlin (Deutsche Texte des Mittelalters 20).

Tertullian: Über den Götzendienst/De Idolatria (1912). In: Tertullians ausgewählte Schriften, Bd. 1: Tertullians private und katechetische Schriften. Neu übersetzt von Karl Adam Heinrich Kellner. Kempten (Bibliothek der Kirchenväter 7), S. 137-174.

\section{Forschungsliteratur}

Belkin, Johanna (1971): Das mechanische Menschenbild in der Floredichtung Konrad Flecks. In: Zeitschrift für deutsches Altertum 100, S. 325-346.

de Bruyne, Edgar (1946 [Reprint Genève 1975]): Etudes d'esthétique médiévale, Bd. 1: De Boèce à Jean Scot Erigène. Bruges.

Fasbender, Christoph (1999): reht alsam er lebte. Nachbildung als Überbietung der Natur in der Epik des Mittelalters. In: Natur und Kultur in der der deutschen Literatur des Mittelalters. Hg. von Alan Robertshaw/Gerhard Wolf in Zusammenarbeit mit Frank Fürbeth/Ulrike Zitzelsperger. Tübingen, S. 53-64.

Kris, Ernst/Otto Kurz (1995): Die Legende vom Künstler. Ein geschichtlicher Versuch, Frankfurt/M.

Niehr, Klaus (2003): ad vivum - al vif. Begriffs- und kunstgeschichtliche Anmerkungen zur Auseinandersetzung mit der Natur in Mittelalter und früher Neuzeit. In: Natur im Mittelalter. Konzeptionen - Erfahrungen - Wirkungen. Akten des 9. Symposiums des Mediävistenverbandes, Marburg 2001. Hg. von Peter Dilg. Berlin, S. 472-487.

Pekáry, Thomas (2002): Imago res mortua est. Untersuchungen zur Ablehnung der bildenden Künste in der Antike. Stuttgart (Heidelberger Althistorische Beiträge und Epigraphische Studien 38).

Roskoff, Gustav: Geschichte des Teufels. Eine kulturhistorische Satanologie von den Anfängen bis ins 18. Jahrhundert. Nördlingen 1987.

Speer, Andreas (1994): Kritische Überlegungen zur mittelalterlichen Ästhetik. In: Ingrid CraemerRugenberg/Andreas Speer (Hg.), Scientia und ars im Hoch- und Spätmittelalter. Berlin, New York 1994, S. 945-966.

Strausberg, Michael (1998): Faszination Zarathustra. Zoroaster und die Europäische Religionsgeschichte der Frühen Neuzeit. 2 Bde. Berlin, New York (Religionsgeschichtliche Versuche und Vorarbeiten 42, 11-2). 